4 of the patients also had a diagnosis of chronic fatigue syndrome (CFS). All patients had tilt tests (HUTT) of which fifteen satisfied criteria 1 and five satisfied criteria 2. The following medications were used:

\begin{tabular}{ll}
\hline Medications & Percentage \\
\hline Slow release sodium chloride & $75 \%$ \\
Beta Blockers & $45 \%$ \\
Fludrocortisone & $55 \%$ \\
Midodrine & $40 \%$ \\
Ivabradine & $20 \%$ \\
Others & $35 \%$ \\
\hline
\end{tabular}

Outcome after medications:

\begin{tabular}{ll}
\hline Outcome & Percentage \\
\hline Significant Improvement & $45 \%$ \\
Moderate Improvement & $45 \%$ \\
No/Mild improvement & $10 \%$ \\
\hline
\end{tabular}

Discussion PoTS is a multisystemic condition presenting typically in the adolescent girls with not many published studies in children. A tilt test helps to confirm the diagnosis and tailored management. Medications helps in symptomatic improvement in the majority with ability to resume education \& activities. Children with deconditioning type of PoTS secondary to CFS often show poor response to medications.

\section{G16(P) CHEST PAIN IN CHILDREN: DOES IT NEED CARDIOLOGY INPUT?}

B Sarder, S Johal, P Nair. Paediatric Department, Bedford Hospital NHS Trust, Bedford, UK

\subsection{6/archdischild-2020-rcpch.6}

Introduction Chest pain in children is a common complaint \& is one of the commonest reasons for referral to a paediatrician with cardiology expertise (PEC) after heart murmurs. Although it causes high levels of anxiety in parents, the causes for these are usually benign. Often these children are referred to PECs for investigation and management.

Aim To look at the demographics of children presenting to the PEC outpatient clinic, review the presenting symptoms, investigations done and diagnosis with outcome.

Methods We reviewed the notes for the patient attending PEC clinic for complaints of chest pain over the last 3 years.

Results There were 40 referrals to the PEC clinic for children with chest pain. The age ranges were between 5-16 years with the average age being 13.8 years, with $55 \%$ being in the 12-16 years range. There were 17 boys and 23 girls. 29 children complained of bilateral, 8 left sided and 3 had central chest pain. 24 of these children had other symptoms like palpitations, dizziness, chest tightness, lethargy, chronic fatigue, breathing difficulties, anxiety, pain in the arms and fingers. All of them had further investigations that included blood tests, ECG, CXR, and echocardiography. Amongst the 26 children who had vitamin D levels done, 38\% had low levels, $42 \%$ had insufficient levels and $20 \%$ had normal levels. All patients had ECGs and echocardiography done which did not reveal any underlying cardiac cause for the chest pain. High dose vitamin D supplements were given for 9 cases. None of the cases needed cardiac interventions or treatment.

Discussion Chest pain is a common problem in adolescent children particularly in the 12-16 age group. Investigations were usually all normal, apart from findings of low vitamin D level in $80 \%$ of the children screened. Notably cardiac investigations were all normal suggesting that the possibility of underlying cardiac problems is very rare. Based on this study we recommend that chest pain in children should be seen in the general paediatric clinic and necessitates cardiac referrals or investigation only in a handful of these.

\section{G17(P) KAWASAKI DISEASE IN A 10 WEEKS OLD INFANT WITH CORONARY DILATATION}

MS Iqbal, AP Singh, E Haworth, S Raina. Department of Paediatrics, The Princess Alexandra Hospital, Harlow, UK

\subsection{6/archdischild-2020-rcpch.7}

10 weeks old infant presented to emergency with 9 days history of fever. He was seen by G.P twice during this illness, initially the impression of viral illness was made and on second time child had 9 days of fever and looked miserable and lethargic so was referred to padiatrics emergency.

There was no significant past medical history. No history of sick contacts and no recent travel. At presentation the child was febrile and irritable with no obvious source for fever. He was started on IV Ceftriaxone and Aciclovir. Initial bloods revealed profound leucocytosis (Total WBC 76, neutrophils 50.1) and CRP of 236, with serial blood films showing a reactive picture and left shift. Chest $\mathrm{x}$-ray was clear, urine/ blood cultures were negative and LP showed 8 WCC with negative cultures. After 72 hours of inpatient care the child was referred to tertiary centre in view persistent fever and irritability.

In the tertiary Hospital, parents told that child had bloodshot eyes, puffy swollen feet and red lips. Child was started on IVIG $2 \mathrm{gm} / \mathrm{kg}$ and high dose aspirin. Child had echocardiography which showed right coronary artery (RCA) dilatation maximal at distal segment measuring $3.58 \mathrm{~mm}$ with $\mathrm{Z}$ score of 8.86. RCA at mid-point and proximal segment measured 3.06 and 2.93 respectively with Z-score of 6.31 and 5.14 respectively. Left anterior descending artery measured 2.42 with Z-score of 4.27 .

During in-patient child was having on-going mild fever and in view of significant coronary dilatation child was started on low molecular weight heparin and also second line treatment with infliximab to which he responded well. Repeat echocardiography showed stable coronary dilatation.

Kawasaki is a mucocutaneous lymph node syndrome with specific diagnostic features, having said that it seems to be a rarity to find all the features consistent with diagnosis. The age presentation, less than 6 month is a very uncommon reported finding in UK and the lack of any diagnostic features initially for Kawasaki except fever lasting for more than 5 days makes this case an important academic and learning standpoint for paediatric trainees. 